\title{
Information Depth and Kikuchi Band Contrast in Electron Backscatter Diffraction Patterns
}

\author{
Aimo Winkelmann \\ Max-Planck-Institut für Mikrostrukturphysik, Weinberg 2, D-06120 Halle (Saale), Germany \\ Electron backscatter diffraction (EBSD) patterns are formed by contributions of electrons that are \\ backscattered from the sample in different depths. The depth range which is relevant for crystal \\ structure information in EBSD is influenced by inelastic and elastic scattering processes of the \\ incident electrons.
}

By dynamical electron diffraction simulations of EBSD patterns [1], the correlation between assumed depth distributions of backscattered electrons and the observed Kikuchi band contrast and sharpness can be analyzed. It is demonstrated how different depth distributions of backscattered electrons produce characteristically different EBSD patterns by comparison with the depthdependent diffracted probability density inside the crystal.

Anomalous absorption of the two different types of Bloch waves in the crystal leads to a characteristic contrast change in EBSD Kikuchi bands when the effective interaction thickness of the outgoing electrons is increased. At low sampling depths, Kikuchi bands show increased intensity in the middle of the band (Fig.1), whereas they show an intensity minimum for thicknesses in which the different absorption coefficients for Bloch waves traveling in a different crystal environment (either on atomic planes or in between them) become relevant (Fig.2).

An additional effect comes into play at very low thicknesses. The dynamical interference effects that set up the Bloch wave fields in the crystal need a certain depth to fully develop. Since the modulation of the backscattered diffraction probability is significantly reduced in this case, a reduced sharpness of the EBSD patterns results (Fig.3).

[1] A. Winkelmann, C. Trager-Cowan, F. Sweeney, A. P. Day, P. Parbrook Ultramicroscopy 107, 414 (2007) 


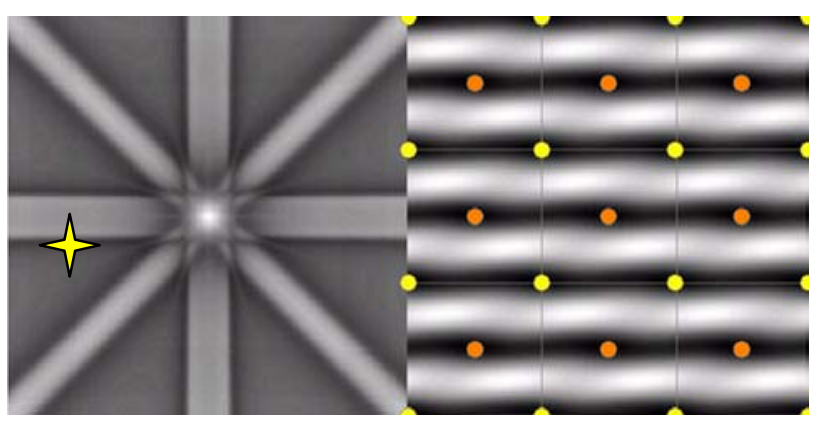

Fig.1: left: Simulated nine-beam EBSD pattern for Fe (001) at $15 \mathrm{keV}$ right: diffracted probability density in the crystal, for the outgoing direction corresponding to the star in the left part of the figure (edge of the horizontal Kikuchi band with an intensity minimum), 3x3 unit cells viewed along the c-axis, constant depth distribution from $0 . .40 \mathrm{~nm}$, inelastic mean free path $8 \mathrm{~nm}$. Outgoing probability density (white) is accumulated between the atomic planes, and electrons backscattered at the atomic positions cannot couple efficiently to the respective outgoing direction, giving a diffraction-induced minimum of backscattered electrons.

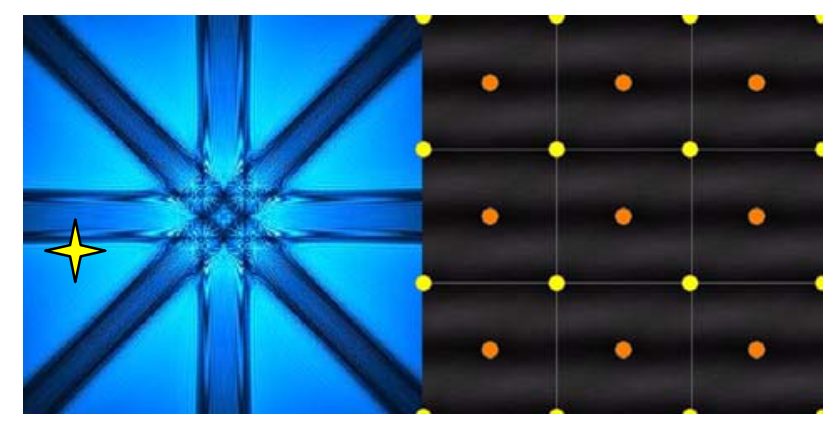

Fig.2: Simulated EBSD pattern and probability distribution as in Fig. 1 for electrons from $85 \ldots 90 \mathrm{~nm}$ depth. The only electrons from greater depths which can survive the inelastic scattering are the ones which travel between the atomic planes. A local minimum in Fig. 1 can turn into a local maximum in Fig. 2.

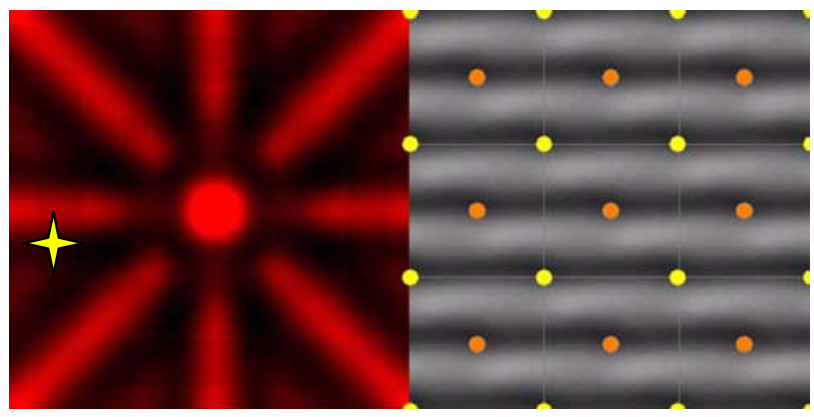

Fig.3: Simulated EBSD pattern as in Fig. 1 for electrons from $0 . .2 \mathrm{~nm}$ depth. The probability distribution shows reduced modulation (right) and the EBSD pattern (left) is less sharp. 